\title{
On the Performance Limits of Pilot-Based Estimation of Bandlimited Frequency-Selective Communication Channels
}

\author{
Francesco Montorsi, Student Member, IEEE, and Giorgio Matteo Vitetta, Senior Member, IEEE
}

\begin{abstract}
In this paper the problem of assessing bounds on the accuracy of pilot-based estimation of a bandlimited frequency selective communication channel is tackled. Mean square error is taken as a figure of merit in channel estimation and a tapped-delay line model is adopted to represent a continuous time channel via a finite number of unknown parameters. This allows to derive some properties of optimal waveforms for channel sounding and closed form Cramér-Rao bounds.
\end{abstract}

Index Terms-Estimation, Fading Channels.

\section{INTRODUCTION}

Channel estimation plays a critical role in modern digital communication systems, where receivers often need to acquire the channel state for each transmitted data packet. To facilitate channel estimation, pilot signals, i.e. waveforms known at the receiver, are usually embedded in the transmitted data signal [1]. In any application, it is important to devise pilot signals in a way that, for a given figure of merit, optimality or near optimality is ensured in a wide range of channel conditions. Important examples of such a figure are represented by the Cramér-Rao bound (CRB) and the Bayesian CRB (BCRB), which limit the mean square error (MSE) performance achievable by any channel estimation algorithm. These bounds have been evaluated for a pilot-aided transmission in single-input multiple-output (SIMO) and multiple-input multiple-output (MIMO) block frequency selective fading scenarios in [2], [3] under the assumptions that: a) the pilot signal is generated by a digital modulator fed by a sequence of pilot data; b) a symbol-spaced discretetime model can be adopted for data transmission and, in particular, for the representation of a multipath fading channel; c) the tap gains of the channel model are independent and identically distributed complex Gaussian random variables (this assumption is made in [3] only).

In this correspondence we revisit the problem of assessing performance limits on pilot-aided channel esti-

F. Montorsi and G. M. Vitetta are with Department of Information Engineering, University of Modena e Reggio Emilia (e-mail: francesco.montorsi@unimore.it and giorgio.vitetta@unimore.it). mation over a frequency selective channel, taking a novel perspective. In fact, we adopt a continuous time (instead of a discrete time) model for the overall description of a channel sounding system and adopt the MSE of the estimated continuous time channel impulse response (CIR) as a figure of merit. Then, we show that bounds for this figure of merit can be derived exploiting CRB's referring to the estimation of the tap gains of a tapped delay line (TDL) model of the communications channel. This sheds new light on both the achieveable limits and the properties of optimal waveforms for channel sounding; in particular, the role played by the properties of a continuous time communication channel in limiting the MSE performance in channel estimation is unveiled.

This Correspondence is organized as follows. In Section [II the model of a system for pilot-based channel estimation is described in detail and two figures of merit for channel estimation are defined. Two bounds on such figures are derived in Section [III and are evaluated in Section IV for two different scenarios. Finally, Section $\mathrm{V}$ offers some conclusions.

\section{Signal And System Models}

In the following we consider the channel sounding system illustrated in Fig. 1. In this system, the transmitter sends a bandlimited real low-pass signal $x(t)$ (dubbed pilot signal in the following), having bandwidth $B$ and known to the receiver, over a frequency selective communication channel characterized by its impulse response $h(t)$ (or, equivalently, by its frequency response $H(f)$ ). Let $r(t)=x(t) \otimes h_{B}(t)+n(t)$ denote the noisy channel response to $x(t)$, where $\otimes$ denotes the convolution operator, $n(t)$ is a complex circularly symmetric additive white Gaussian noise (AWGN) characterized by a twosided power spectral density $2 N_{0}$ and

$$
h_{B}(t) \triangleq \int_{-B}^{B} H(f) \exp (j 2 \pi f t) d f
$$

is a bandlimited version of $h(t)$; note that $h_{B}(t)$ fully describes the noiseless channel behavior in the time domain for any input signal whose bandwidth 


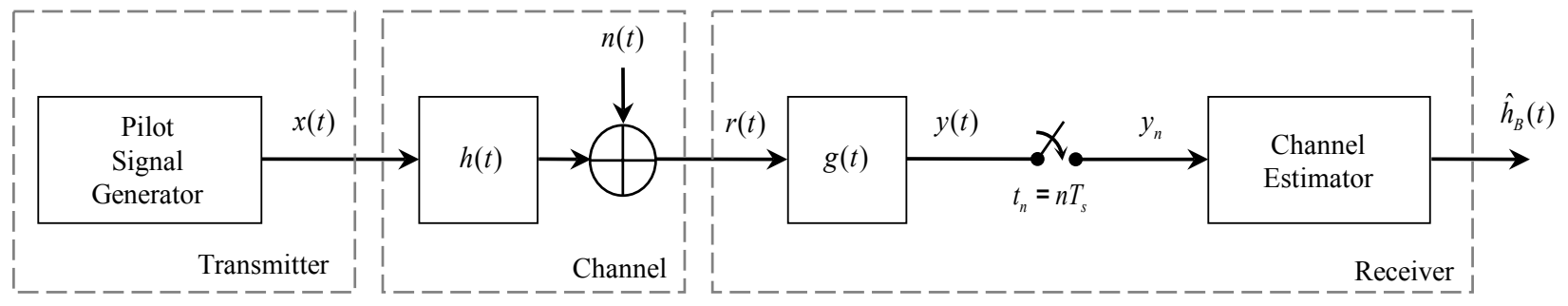

Figure 1. Channel sounding system: baseband model.

does not exceed $B$. The noisy signal $r(t)$ feeds a receiver which accomplishes ideal low-pass filtering (with bandwidth $B$ ), followed by sampling at a frequency $f_{s}=1 / T_{s}=2 B$, where $T_{s}$ denotes the sampling period (in Fig. $11 t_{n} \triangleq n T_{s}$ represents the $n$th sampling instant). We assume that the impulse response of the low-pass filter is $g(t)=2 B \operatorname{sinc}(2 B t)$, so that its frequency response takes on a unitary value in the frequency interval $(-B, B)$; then, the filter response $y(t)$ is given by

$$
y(t)=x(t) \otimes h_{B}(t)+w(t),
$$

where $w(t)$ is complex bandlimited Gaussian process having zero mean and a two-sided power spectral density $S_{w}(f)=2 N_{0}$ for $|f|<B$ and zero elsewhere; note that its autocorrelation function is $R_{w}(\tau)=4 N_{0} B \operatorname{sinc}(2 B \tau)$ and its average statistical power is $\sigma_{w}^{2}=R_{w}(0)=4 N_{0} B$. Sampling $y(t)$ generates the sequence $\left\{y_{n} \triangleq y\left(t_{n}\right)\right\}$, which feeds a channel estimator. This processes a finite subset of elements of $\left\{y_{n}\right\}$ to generate an estimate $\hat{h}_{B}(t)$ of $h_{B}(t)$. It is important to point out that:

1. Any channel estimation algorithm assumes a specific parametric representation of the communication channel. In the following, we adopt the well known tapped delay line (TDL) model for a bandlimited communication channel [4] and assume a finite memory (i.e., a finite number of active taps); for this reason, $h_{B}(t)$ is expressed as

$$
h_{B}(t) \cong 2 B \sum_{l=-L_{1}}^{L_{2}} h_{B, l} \operatorname{sinc}\left(2 B\left(t-\frac{l}{2 B}\right)\right),
$$

where

$$
\begin{aligned}
h_{B, l} & \triangleq \frac{1}{2 B} h_{B}\left(t_{l}\right)=\frac{1}{2 B} h_{B}\left(\frac{l}{2 B}\right) \\
& =\frac{1}{2 B} \int_{-B}^{B} H(f) \exp \left(j 2 \pi l \frac{f}{2 B}\right) d f
\end{aligned}
$$

for any $l$ and $L_{1}, L_{2}>0$ (the overall number of active taps 11 is $\left.L \triangleq L_{1}+L_{2}+1\right)$.

2 . For a given sounding waveform $x(t)$, a measure of the accuracy of the channel estimate $\hat{h}_{B}(t)$ is provided by the MSE, defined as

$$
\begin{aligned}
\varepsilon_{B, L} & \triangleq \frac{1}{2 B} \mathbb{E}_{w}\left\{\int_{-\infty}^{+\infty}\left|e_{B, L}(t)\right|^{2} d t\right\} \\
& =\frac{1}{2 B} \mathbb{E}_{w}\left\{\int_{-B}^{B}\left|E_{B, L}(f)\right|^{2} d f\right\},
\end{aligned}
$$

where $e_{B, L}(t) \triangleq h_{B}(t)-\hat{h}_{B}(t)$ and $E_{B, L}(f)$ $\triangleq H_{B}(f)-\hat{H}_{B}(f)$, if the CIR $h_{B}(t)$ is modelled as a deterministic unknown function, and as

$$
\begin{aligned}
\bar{\varepsilon}_{B, L} & \triangleq \frac{1}{2 B} \mathbb{E}_{w, h_{B}}\left\{\int_{-\infty}^{+\infty}\left|e_{B, L}(t)\right|^{2} d t\right\} \\
& =\frac{1}{2 B} \mathbb{E}_{w, h_{B}}\left\{\int_{-B}^{B}\left|E_{B, L}(t)\right|^{2} d f\right\},
\end{aligned}
$$

if $h_{B}(t)$ is modelled as an unknown random process. Here, $H_{B}(f)\left(\hat{H}_{B}(f)\right)$ denotes the Fourier continuous transform of $h_{B}(t)\left(\hat{h}_{B}(t)\right)$ and $\mathbb{E}_{X}\{\cdot\}$ denotes a statistical average with respect to the random parameter $X$.

Substituting (3) in (2) yields

$$
y(t)=\sum_{l=-L_{1}}^{L_{2}} h_{B, l} x\left(t-\frac{l}{2 B}\right)+w(t),
$$

so that the sample $y_{n}$ can be expressed as $y_{n} \triangleq$ $y\left(n T_{s}\right)=y\left(\frac{n}{2 B}\right)=\sum_{l=-L_{1}}^{L_{2}} h_{B, l} x_{n-l}+w_{n}$, where $x_{n} \triangleq x\left(t_{n}\right)$ and $w_{n} \triangleq w\left(t_{n}\right)$. In our system model, the channel estimator processes the set of $N$ consecutive noisy samples $\left\{y_{n}, n=1,2, \ldots, N\right\}$, i.e. the noisy vector $\mathbf{y} \triangleq\left[y_{1}, y_{2}, \ldots, y_{N}\right]^{T}$, to generate an estimate $\hat{\mathbf{h}}_{B} \triangleq\left[\hat{h}_{B,-L_{1}}, \hat{h}_{B, 1-L_{1}}, \ldots, \hat{h}_{B, L_{2}}\right]^{T}$ of the $L$ dimensional channel parameter vector $\mathbf{h}_{B} \triangleq\left[h_{B,-L_{1}}\right.$, $\left.h_{B, 1-L_{1}}, \ldots, h_{B, L_{2}}\right]^{T}$. This results in the estimated CIR

${ }^{1}$ Note that the values of the parameters $L_{1}$ and $L_{2}$ (and, consequently, the value of $L$ ) should be large enough to ensure a good accuracy in the representation of the bandlimited CIR $h_{B}(t)$ and, in particular, to capture most of the energy of this signal. For this reason, such values mainly depend on the power delay profile (PDP) of the considered channel and are not necessarily equal (further details are provided in Section IV. 
$\hat{h}_{B}(t) \triangleq 2 B \sum_{l=-L_{1}}^{L_{2}} \hat{h}_{B, l} \operatorname{sinc}\left(2 B\left(t-\frac{l}{2 B}\right)\right)$. It is easy to show that: a) $\mathbf{y}$ can be put in matrix form as

$$
\mathbf{y}=\mathbf{X} \mathbf{h}_{B}+\mathbf{w}
$$

where $\mathbf{w}=\left[w_{1}, w_{2}, \ldots, w_{N}\right]^{T}$ is a vector of independen $\sqrt{2}$ and identically distributed complex Gaussian random variables (each having zero mean and variance $\sigma_{w}^{2}=4 N_{0} B$ ) and $\mathbf{X}$ is a $N \times L$ matrix whose element on its $i$-th row and $j$-th column is $X_{i, j}=x_{i+j+L_{1}-1}$ (with $i=1,2, \ldots, N$ and $j=-L_{1}, 1-L_{1}, \ldots, L_{2}$ ); b) thanks to the property of orthogonality of the sinc $(\cdot)$ functions appearing in the channel model (3), the MSE (4) can be also expressed as

$\varepsilon_{B, L}=\sum_{l=-L_{1}}^{L_{2}} \mathbb{E}_{w}\left\{\left|h_{B, l}-\hat{h}_{B, l}\right|^{2}\right\}=\sum_{l=-L_{1}}^{L_{2}} \operatorname{MSE}\left(\hat{h}_{B, l}\right)$,

i.e. as a scaled sum of the MSE errors associated with the $L$ channel taps (a similar expression can be developed for $\bar{\varepsilon}_{B, L}$ (5)). In the following Section the problem of deriving bounds for the parameters $\varepsilon_{B, L}$ (4) and $\bar{\varepsilon}_{B, L}$ (5) is tackled.

\section{Evaluation of PeRformance Limits ON CHANNEL ESTIMATION}

In estimating the vector $\mathbf{h}_{B}$ defined in previous Section, it can be modelled as a vector of unknown deterministic parameters or as a vector of random parameters with given statistical properties. In this Section we take into consideration both models, deriving some new bounds on the channel estimation accuracy.

\section{A. CRB-based performance limit}

In this Paragraph we focus on the class of unbiased estimators of the unknown deterministic vector $\mathbf{h}_{B}$ and derive a lower bound for the parameter $\varepsilon_{B, L}$ (7). To begin, we note that $\varepsilon_{B, L}$ can be evaluated as $\varepsilon_{B, L}=\sum_{l=-L_{1}}^{L_{2}} \operatorname{var}\left(\hat{h}_{B, l}\right)$, since $\mathbb{E}_{w}\left\{\left|h_{B, l}-\hat{h}_{B, l}\right|^{2}\right\}=\operatorname{var}\left(\hat{h}_{B, l}\right)$ (with $l=-L_{1}, 1-L_{1}$, $\ldots, L_{2}$ ), where $\operatorname{var}(X)$ denotes the variance of the random variable $X$. A lower bound to $\operatorname{var}\left(\hat{h}_{B, l}\right)$ for the above mentioned class of estimators is represented by the CRB [5], which, in this case, can be expressed as 3 $\operatorname{var}\left(\hat{h}_{B, l}\right) \geq\left[\mathbf{J}_{C}^{-1}\left(\mathbf{h}_{B}\right)\right]_{l, l}$ with $l=-L_{1}, 1-L_{1}, \ldots, L_{2}$, where

\footnotetext{
${ }^{2}$ The independence of noisy samples is due to the fact that $\mathrm{E}\left\{w_{l} w_{k}^{*}\right\}=R_{w}\left(t_{l}-t_{k}\right)=4 N_{0} B \operatorname{sinc}\left(2 B\left(t_{l}-t_{k}\right)\right)=$ $4 N_{0} B \operatorname{sinc}(l-k)=0$ if $l \neq k$. In other words, noise samples are uncorrelated and, being jointly Gaussian random variables, are statistically independent.

${ }^{3}$ Note that, to ease the reading, the indices of the rows and of the columns of $\mathbf{J}_{C}\left(\mathbf{h}_{B}\right)$ and $\mathbf{J}_{C}^{-1}\left(\mathbf{h}_{B}\right)$ range from $-L_{1}$ to $L_{2}$.
}

$$
\begin{aligned}
& {\left[\mathbf{J}_{C}\left(\mathbf{h}_{B}\right)\right]_{l, p} \triangleq} \\
& \left.\quad \mathbb{E}_{\mathbf{y}}\left\{\frac{\partial \ln f_{\mathbf{y}}\left(\mathbf{y} ; \tilde{\mathbf{h}}_{B}\right)}{\partial \tilde{h}_{B, l}^{*}}\left(\frac{\partial \ln f_{\mathbf{y}}\left(\mathbf{y} ; \tilde{\mathbf{h}}_{B}\right)}{\partial \tilde{h}_{B, p}^{*}}\right)^{*}\right\}\right|_{\tilde{\mathbf{h}}_{B}=\mathbf{h}_{B}}
\end{aligned}
$$

with $l, p=-L_{1}, 1-L_{1}, \ldots, L_{2}$, is an $L \times L$ complex matrix, known as Fisher Information Matrix (FIM), $f_{\mathbf{y}}\left(\mathbf{y} ; \mathbf{h}_{B}\right)$ is the joint probability density function of y (6) parameterized by the unknown (random) vector $\mathbf{h}_{B}$ and $\tilde{\mathbf{h}}_{B} \triangleq\left[\tilde{h}_{B,-L_{1}}, \tilde{h}_{B, 1-L_{1}}, \ldots, \tilde{h}_{B, L_{2}}\right]^{T}$ is a (deterministic) trial vector $]$. Then, the lower bound

$$
\varepsilon_{B, L} \geq \sum_{l=-L_{1}}^{L_{2}}\left[\mathbf{J}_{C}^{-1}\left(\mathbf{h}_{B}\right)\right]_{l, l}=\operatorname{tr}\left(\mathbf{J}_{C}^{-1}\left(\mathbf{h}_{B}\right)\right)
$$

can be formulated for $\varepsilon_{B, L}$, where $\operatorname{tr}(\mathbf{A})$ denotes the trace of a square matrix A. From the model (6) it can be easily inferred that, given $\mathbf{h}_{B}=\tilde{\mathbf{h}}_{B}, \mathbf{y} \sim \mathcal{C N}\left(\mu, \mathbf{R}_{\mathbf{w}}\right)$, where $\mu \triangleq \mathbf{X} \tilde{\mathbf{h}}_{B}$ and $\mathbf{R}_{\mathbf{w}}=\sigma_{w}^{2} \mathbf{I}_{N}$ is the covariance matrix of $\mathbf{w}\left(\mathbf{I}_{N}\right.$ is the $N \times N$ identity matrix), so that the element on $l$-th row and $p$-th column of $\mathbf{J}_{C}\left(\mathbf{h}_{B}\right)$ can be expressed as (e.g. see [6, Paragraph 2], [7, rel. B.3.25])

$$
\begin{aligned}
{\left[\mathbf{J}_{C}\left(\mathbf{h}_{B}\right)\right]_{l, p}=} & 2 \operatorname{Re}\left[\left(\frac{\partial \mu}{\partial \tilde{h}_{B, l}^{*}}\right)^{H} \mathbf{R}_{\mathbf{w}}^{-1} \frac{\partial \mu}{\partial \tilde{h}_{B, p}^{*}}\right]_{\tilde{\mathbf{h}}_{B}=\mathbf{h}_{B}} \\
& +\left.\operatorname{tr}\left(\mathbf{R}_{\mathbf{w}}^{-1} \frac{\partial \mathbf{R}_{\mathbf{w}}}{\partial \tilde{h}_{B, l}^{*}} \mathbf{R}_{\mathbf{w}}^{-1} \frac{\partial \mathbf{R}_{\mathbf{w}}}{\partial \tilde{h}_{B, p}^{*}}\right)\right|_{\tilde{\mathbf{h}}_{B}=\mathbf{h}_{B}}
\end{aligned}
$$

with $l, p=-L_{1}, 1-L_{1}, \ldots, L_{2}$, where $\operatorname{Re}(x)$ denotes the real part of a complex number $x$. It is easy to show that $\partial \mu / \partial \tilde{h}_{B, p}^{*}=(1 / 2)(1+j)\left[x_{1-p}, x_{2-p}, \ldots, x_{N-p}\right]^{T}$, where $\operatorname{Im}(x)$ denotes the imaginary part of a complex number $x$. Then, substituting this result in (10) and keeping into account that $\partial \mathbf{R}_{\mathrm{w}} / \partial \tilde{h}_{l}^{*}=\mathbf{0}_{N}$ (where $\mathbf{0}_{N}$ denotes the $N \times N$ null matrix) yields, after some manipulation, the expression

$$
\begin{aligned}
{\left[\mathbf{J}_{C}\left(\mathbf{h}_{B}\right)\right]_{l, p} } & =\frac{1}{\sigma_{w}^{2}} \operatorname{Re}\left\{\sum_{m=1}^{N} x_{m-l}^{*} x_{m-p}\right\} \\
& =\frac{N}{\sigma_{w}^{2}} \operatorname{Re}\left\{\frac{1}{N} \sum_{k=1-p}^{N-p} x_{k}^{*} x_{k+p-l}\right\} .
\end{aligned}
$$

\footnotetext{
${ }^{4}$ The trial vector is used to indicate that the differentiation operation in the FIM definition is against a deterministic (versus random) complex variable. In particular, if $f(\boldsymbol{\mu})$ is some function of the deterministic complex vector $\boldsymbol{\mu}=\left[\ldots, \mu_{i}, \ldots\right]$, then the usual definition $\frac{\partial f(\boldsymbol{\mu})}{\partial \mu_{i}^{*}} \triangleq \frac{1}{2}\left(\frac{\partial f(\boldsymbol{\mu})}{\partial \operatorname{Re}\left\{\mu_{i}\right\}}+j \frac{\partial f(\boldsymbol{\mu})}{\partial \operatorname{Im}\left\{\mu_{i}\right\}}\right)$ applies.
} 
The last result shows that the FIM depends on the sample sequence $\left\{x_{k}\right\}$ of the channel sounding waveform $x(t)$, but is not influenced by the parameters of the TDL channel model. We are interested in optimizing the lower bound (9) (i.e., in minimizing its right hand side) with respect to such a waveform. To tackle this optimization problem we assume that $x(t)$ is a sample function of a bandlimited random process having the following properties: a) it is wide sense stationary (WSS); b) it has zero mean and power spectral density (PSD) $S_{x}(f)>0(=0)$ for $f \in(-B, B)(f \notin(-B, B))$; c) its autocorrelation function $R_{x}(\tau)$ tends to 0 for $\tau \rightarrow \infty$ more quickly than $1 / \tau ; \mathrm{d})$ it is ergodic in autocorrelation. These assumptions entail that: 1) the sample sequence $\left\{x_{n} \triangleq x\left(t_{n}\right)\right\}$ is a discrete-time WSS random process having zero mean, autocorrelation function $R_{x}[l]=R_{x}\left(l T_{s}\right)$ and power spectral density

$$
\begin{aligned}
\bar{S}_{x}(f) & =\sum_{k=-\infty}^{+\infty} R_{x}[k] \exp \left(-j 2 \pi f k T_{s}\right) \\
& =f_{s} \sum_{l=-\infty}^{+\infty} S_{x}\left(f-l f_{s}\right) ;
\end{aligned}
$$

2) $R_{x}[l]$ decreases more quickly than $1 / l$ for $l \rightarrow \infty$, so that the series $\sum_{l=-\infty}^{\infty}\left|R_{x}[l]\right|$ is convergent; 3$)\left\{x_{n}\right\}$ is ergodic in autocorrelation. Under the above assumptions, the equality $\lim _{N \rightarrow \infty} \frac{1}{N} \sum_{k=1-p}^{N-p} x_{k}^{*} x_{k+p-l}=R_{x}[p-l]$ holds with unit probability (see (11)), so that for a finite (and large) $N$ (i.e., when a large number of samples of the received signal is available for channel estimation) the element $\left[\mathbf{J}_{C}\left(\mathbf{h}_{B}\right)\right]_{l, p}$ (11) can be approximated as

$$
\left[\mathbf{J}_{C}\left(\mathbf{h}_{B}\right)\right]_{l, p} \cong \frac{N}{\sigma_{w}^{2}} R_{x}[p-l] .
$$

The adoption of this approximation leads to a real symmetric Toeplitz FIM; this implies that: a) any eigenvalue of $\mathbf{J}_{C}^{-1}\left(\mathbf{h}_{B}\right)$ is always not smaller than $\inf \left(\bar{S}_{x}(f)\right)$ [8, lemma 4.1], so that $\operatorname{tr}\left(\mathbf{J}_{C}^{-1}\left(\mathbf{h}_{B}\right)\right)$ (see (9)) grows unlimitedly as $L \rightarrow \infty$ (this means that, for a given $N$, as the number $L$ of channel parameters to be estimated increases, the overall MSE diverges); b) the following asymptotic result holds [8, theorem 5.2c]:

$$
\lim _{L \rightarrow \infty} \frac{1}{L} \operatorname{tr}\left(\mathbf{J}_{C}^{-1}\left(\mathbf{h}_{B}\right)\right)=T_{s} \frac{\sigma_{w}^{2}}{N} \int_{-f_{s} / 2}^{f_{s} / 2} \frac{d f}{\bar{S}_{x}(f)},
$$

since $N \bar{S}_{x}(f) / \sigma_{w}^{2}$ belongs to the Wiener class (i.e., the sum of the absolute values of the FIM diagonal elements remains bounded as $L \rightarrow \infty$; in other words $\left(N / \sigma_{w}^{2}\right)$ $\left.\sum_{l=-\infty}^{+\infty}\left|R_{x x}[l]\right|<\infty\right), \bar{S}_{x}(f)$ is a real valued function and $\bar{S}_{x}(f)>0$ for any $f$. Then, from (9) and (14) the lower bound

$$
\lim _{L \rightarrow \infty} \frac{\varepsilon_{B, L}}{L} \geq T_{s} \frac{\sigma_{w}^{2}}{N} \int_{-f_{s} / 2}^{f_{s} / 2} \frac{d f}{\bar{S}_{x}(f)}
$$

can be easily inferred. This result depends on the power spectrum $\bar{S}_{x}(f)$, which can be optimized to improve the quality of channel estimation under the constraint $T_{s} \int_{-f_{s} / 2}^{f_{s} / 2} \bar{S}_{x}(f) d f=P_{x}$ on the average statistical power $P_{x}$ of $\left\{x_{n}\right\}$. Applying the method of Lagrange multipliers to this optimization problem leads to the conclusion that the right hand side of (15) is maximised (under the given constraint) if $\bar{S}_{x}(f)=P_{x}$ for any $f \in\left(-f_{s} / 2, f_{s} / 2\right)$, i.e. if the power spectrum of $\left\{x_{n}\right\}$ is uniform (equivalently, $R_{x}[l]=P_{x} \delta[l]$ ); this occurs if (see (12))

$$
S_{x}(f)=\left\{\begin{array}{ll}
\frac{\bar{S}_{x}(f)}{f_{s}}=\frac{P_{\alpha}}{f_{s}}=\frac{P_{x}}{2 B} & f \in\left(-f_{s} / 2, f_{s} / 2\right) \\
0 & \text { elsewhere }
\end{array},\right.
$$

since $x(t)$ is bandlimited to $f_{s} / 2=B \mathrm{~Hz}$. It is important to note that, if the optimal power spectrum is selected for $\left\{x_{n}\right\}$ and the approximation (13) is used, (13) gives $\left[\mathbf{J}_{C}\left(\mathbf{h}_{B}\right)\right]_{l, p}=\left(N P_{x} / \sigma_{w}^{2}\right) \delta[p-l]$ and the FIM $\mathbf{J}_{C}\left(\mathbf{h}_{B}\right)$ can be put in the form

$$
\mathbf{J}_{C}\left(\mathbf{h}_{B}\right)=\frac{N P_{x}}{\sigma_{w}^{2}} \mathbf{I}_{L},
$$

so that $\mathbf{J}_{C}^{-1}\left(\mathbf{h}_{B}\right)=\left(\sigma_{w}^{2} /\left(N P_{x}\right)\right) \mathbf{I}_{L}$ and $\operatorname{var}\left(\hat{h}_{l}\right) \geq$ $\left[\mathbf{J}_{C}^{-1}\left(\mathbf{h}_{B}\right)\right]_{l, l}=\frac{1}{N} \frac{\sigma_{w}^{2}}{P_{x}}=\frac{1}{N \cdot \mathrm{SNR}}$, where $\mathrm{SNR} \triangleq P_{x} / \sigma_{w}^{2}$ is the signal-to-noise ratio, and the bound (9) becomes

$$
\varepsilon_{B, L} \geq \frac{L}{N \cdot \mathrm{SNR}} \triangleq \beta_{B, L}
$$

This results evidences that, for a given SNR and a given number $N$ of processed samples, an increase in the number $L$ of significant CIR taps is expected to have a negative impact on the quality of CIR estimates. Finally, it's worth noting that the result expressed by (17) is similar to that derived in [2, Paragraph 3.1] for channel estimation based on a training sequence that consists of a large number of uncorrelated channel symbols. In [2, Paragraph 3.1], however, a discrete-time communication model is assumed in the derivation of Cramer-Rao bounds.

\section{B. BCRB-based performance limits}

In this Paragraph we assume uncorrelated scattering (US) and model the CIR $h_{B}(t)$ as a complex Gaussian process characterized by a zero mean (i.e., Rayleigh fading is assumed) and a PDP $P_{h}(\tau)$ with $\int_{-\infty}^{+\infty} P_{h}(\tau) d \tau=1$. Then, we have that $\mathbf{h}_{B} \sim \mathcal{C N}\left(\mathbf{0}_{L}\right.$, 
$\mathbf{R}_{\mathbf{h}}$ ), where $\mathbf{R}_{\mathbf{h}}$ is the covariance matrix of $\mathbf{h}_{B}$; the element on $l$-th row and $p$-th column of $\mathbf{R}_{\mathbf{h}}$ is given by (see (10)

$$
\begin{gathered}
\mathbb{E}\left\{h_{B, l} h_{B, p}^{*}\right\}=\mathbb{E}\left\{\frac{1}{2 B} \int_{-B}^{B} H\left(f_{1}\right) e^{j 2 \pi l \frac{f_{1}}{2 B}} d f_{1}\right. \\
\left.\cdot \frac{1}{2 B} \int_{-B}^{B} H^{*}\left(f_{2}\right) e^{-j 2 \pi p \frac{f_{2}}{2 B}} d f_{2}\right\}= \\
\frac{1}{(2 B)^{2}} \int_{f_{2}=-B}^{B}\left[\int_{f=-B-f_{2}}^{B-f_{2}} R_{H}(f) e^{j 2 \pi l \frac{f}{2 B}} d f\right] e^{j 2 \pi \frac{(l-p) f_{2}}{2 B}} d f_{2}
\end{gathered}
$$

with $l, p=-L_{1}, \quad 1-L_{1}, \ldots, L_{2}$, where $R_{H}(f) \triangleq \mathbb{E}\left\{H\left(f_{0}+f\right) H^{*}\left(f_{0}\right)\right\}$ is the channel autocorrelation function (i.e., the inverse continuous Fourier transform of $P_{h}(\tau)$ ) and $f_{0}$ is an arbitrary frequency. Note that for $l=p$ (19) yields

$$
\begin{aligned}
& \mathbb{E}\left\{\left|h_{B, l}\right|^{2}\right\}= \\
& \quad \frac{1}{(2 B)^{2}} \int_{f_{2}=-B}^{B}\left[\int_{f=-B-f_{2}}^{B-f_{2}} R_{H}(f) e^{j 2 \pi l \frac{f}{2 B}} d f\right] d f_{2} \\
& \quad=\int_{y=-1 / 2}^{-1 / 2}\left[\int_{x=-1 / 2-y}^{1 / 2-y} R_{H}(2 B x) e^{j 2 \pi l x} d x\right] d y
\end{aligned}
$$

Generally speaking, channel estimation algorithms can benefit from the availability of information about channel statistics to improve the quality of their CIR estimate. For such algorithms a lower bound to their MSE performance is provided by the BCRB [9, p. 957-958], which establishes that $\operatorname{MSE}\left(\hat{h}_{B, l}\right) \geq\left[\mathbf{J}_{B}^{-1}\left(\mathbf{h}_{B}\right)\right]_{l, l}$ with $l=-L_{1}$, $1-L_{1}, \ldots, L_{2}$, where $\mathbf{J}_{B}\left(\mathbf{h}_{B}\right)$ is an $L \times L$ complex matrix, known as Bayesian Fisher Information Matrix (BFIM). The element on the $l$-th row and $p$-th column of $\mathbf{J}_{B}(\mathbf{h})$ can be evaluated as [3, equ. 53]

$$
\left[\mathbf{J}_{B}\left(\mathbf{h}_{B}\right)\right]_{l, p}=\left[\mathbf{J}_{C}\left(\mathbf{h}_{B}\right)\right]_{l, p}+\left[\mathbf{J}_{h}\left(\mathbf{h}_{B}\right)\right]_{l, p}
$$

where $\mathbf{J}_{C}\left(\mathbf{h}_{B}\right)$ is the CRB FIM evaluated in the previous Paragraph and

$$
\begin{aligned}
& {\left[\mathbf{J}_{h}\left(\mathbf{h}_{B}\right)\right]_{l, p} \triangleq} \\
& \left.\quad \mathbb{E}_{\mathbf{h}_{B}}\left\{\frac{\partial \ln f_{\mathbf{h}_{B}}\left(\tilde{\mathbf{h}}_{B}\right)}{\partial \tilde{h}_{B, l}^{*}}\left(\frac{\partial \ln f_{\mathbf{h}_{B}}\left(\tilde{\mathbf{h}}_{B}\right)}{\partial \tilde{h}_{B, p}^{*}}\right)^{*}\right\}\right|_{\tilde{\mathbf{h}}_{B}=\mathbf{h}_{B}} .
\end{aligned}
$$

where $f_{\mathbf{h}_{B}}\left(\tilde{\mathbf{h}}_{B}\right)$ denotes the joint pdf of $\mathbf{h}_{B}$. Like in the previous case (see (7) and (9)) the bound

$$
\begin{aligned}
\bar{\varepsilon}_{B, L} & =\sum_{l=-L_{1}}^{L_{2}} \mathbb{E}_{w, h_{B, l}}\left\{\left|h_{B, l}-\hat{h}_{B, l}\right|^{2}\right\} \geq \\
& \sum_{l=-L_{1}}^{L_{2}}\left[\mathbf{J}_{B}^{-1}\left(\mathbf{h}_{B}\right)\right]_{l, l}=\operatorname{tr}\left(\mathbf{J}_{B}^{-1}\left(\mathbf{h}_{B}\right)\right) \triangleq \bar{\beta}_{B, L}
\end{aligned}
$$

\footnotetext{
${ }^{5}$ The eigenvalues of the covariance matrix $\mathbf{R}_{\mathbf{h}}$ are always positive; this implies that the eigenvalues of the matrix $\mathbf{R}_{\mathbf{h}}^{-1}$ are also positive.
}

can easily be developed for $\bar{\varepsilon}_{B, L}$ (5). To evaluate the right hand side of the last inequality, let us compute now the partial derivatives appearing in (22). It is easy to show that

$$
\begin{aligned}
\frac{\partial \ln f_{\mathbf{h}_{B}}\left(\tilde{\mathbf{h}}_{B}\right)}{\partial \tilde{h}_{B, p}^{*}}= \\
-\frac{1}{2}\left(\frac{\partial\left(\tilde{\mathbf{h}}_{B}^{H} \mathbf{R}_{\mathbf{h}}^{-1} \tilde{\mathbf{h}}_{B}\right)}{\partial \operatorname{Re}\left\{\tilde{h}_{B, p}\right\}}+j \frac{\partial\left(\tilde{\mathbf{h}}_{B}^{H} \mathbf{R}_{\mathbf{h}}^{-1} \tilde{\mathbf{h}}_{B}\right)}{\partial \operatorname{Im}\left\{\tilde{h}_{B, p}\right\}}\right)= \\
-\left[\mathbf{R}_{\mathbf{h}}^{-1} \tilde{\mathbf{h}}_{B}\right]_{p} \cdot
\end{aligned}
$$

Then, substituting (24) in (22) yields

$$
\begin{aligned}
\mathbf{J}_{h}\left(\mathbf{h}_{B}\right) & =\mathbb{E}_{\mathbf{h}_{B}}\left\{\left.\left(\mathbf{R}_{\mathbf{h}}^{-1} \tilde{\mathbf{h}}_{B}\right)\left(\mathbf{R}_{\mathbf{h}}^{-1} \tilde{\mathbf{h}}_{B}\right)^{H}\right|_{\tilde{\mathbf{h}}_{B}=\mathbf{h}_{B}}\right\} \\
& =\left(\mathbf{R}_{\mathbf{h}}^{-1}\right)^{H}=\mathbf{R}_{\mathbf{h}}^{-1},
\end{aligned}
$$

since $\mathbf{R}_{\mathbf{h}}$ is an Hermitian matrix. Like the CRB, the BCRB is influenced by the choice of the sounding waveform through $\mathbf{J}_{C}\left(\mathbf{h}_{B}\right)$ (see (21)); in the following a uniform power spectrum is assumed for this waveform (see (16)). Then, substituting (17) and (25) in (21) yields

$$
\begin{aligned}
\mathbf{J}_{B}\left(\mathbf{h}_{B}\right) & =\frac{N P_{x}}{\sigma_{w}^{2}} \mathbf{I}_{L}+\mathbf{R}_{\mathbf{h}}^{-\mathbf{1}} \\
& =N \cdot \operatorname{SNR}\left(\mathbf{I}_{L}+\frac{\mathbf{R}_{\mathbf{h}}^{-\mathbf{1}}}{N \cdot \mathrm{SNR}}\right) .
\end{aligned}
$$

Unluckily, $\mathbf{J}_{B}\left(\mathbf{h}_{B}\right)$ is not a Toepliz matrix and, as far as we know, no asymptotic result is available for the trace of its inverse. However, a simple expression for this trace can be derived if the Taylor series representation

$$
\mathbf{J}_{B}^{-1}\left(\mathbf{h}_{B}\right)=\frac{1}{N \cdot \mathrm{SNR}} \sum_{k=0}^{\infty}\left(-\frac{\mathbf{R}_{\mathbf{h}}^{-1}}{N \cdot \mathrm{SNR}}\right)^{k}
$$

can be adopted for $\mathbf{J}_{B}^{-1}\left(\mathbf{h}_{B}\right)$; this holds if the $L$ eigenvalues of the matrix $(1 /(N \cdot \mathrm{SNR})) \mathbf{R}_{\mathbf{h}}^{-1}$ are distinct and their values are less than unity 5 , i.e. $1 /\left(N \cdot \operatorname{SNR} \cdot \lambda_{i}\right)<1$ (or, equivalently, $\lambda_{i}>\frac{1}{N \cdot \text { SNR }}>0$ ) for $i=1,2, \ldots, L$, where $\left\{\lambda_{i}, i=1,2, \ldots, L\right\}$ denote the (real) eigenvalues of $\mathbf{R}_{\mathbf{h}}$. In fact, this representation entails that

$$
\operatorname{tr}\left\{\mathbf{J}_{B}^{-1}\left(\mathbf{h}_{B}\right)\right\}=\frac{1}{N \cdot \mathrm{SNR}} \sum_{k=0}^{\infty} \operatorname{tr}\left\{\left(-\frac{\mathbf{R}_{\mathbf{h}}^{-\mathbf{1}}}{N \cdot \mathrm{SNR}}\right)^{k}\right\} .
$$


Since $\mathbf{R}_{\mathbf{h}}$ is an hermitian matrix, its inverse $\mathbf{R}_{\mathbf{h}}^{-\mathbf{1}}$ can be factored as $\mathbf{R}_{\mathbf{h}}^{-1}=\mathbf{U} \boldsymbol{\Sigma}^{-1} \mathbf{U}^{H}$ [10, p. 245, sec. 5.2], where $\mathbf{U}$ is a $L \times L$ unitary matrix (whose columns are the eigenvectors of $\mathbf{R}_{\mathbf{h}}$ ) and $\boldsymbol{\Sigma}=\operatorname{diag}\left\{\lambda_{1}, \lambda_{2}, \ldots, \lambda_{L}\right\}$. Exploiting this factorisation it can be easily shown that

$$
\begin{aligned}
\operatorname{tr}\left\{\left(-\frac{\mathbf{R}_{\mathbf{h}}^{-\mathbf{1}}}{N \cdot \mathrm{SNR}}\right)^{k}\right\} & =\operatorname{tr}\left\{\left(\frac{-1}{N \cdot \mathrm{SNR}} \boldsymbol{\Sigma}^{-\mathbf{1}}\right)^{k}\right\} \\
& =\left(\frac{-1}{N \cdot \mathrm{SNR}}\right)^{k} \sum_{i=1}^{L} \frac{1}{\lambda_{i}^{k}}
\end{aligned}
$$

since $\operatorname{tr}\left\{\mathbf{U} \mathbf{D} \mathbf{U}^{H}\right\}=\operatorname{tr}\{\mathbf{D}\}$ for any matrix $\mathbf{D}$ (this result is known as similarity invariance property of the trace operator). Then, substituting the last result in (28) yields

$$
\begin{aligned}
\operatorname{tr}\left(\mathbf{J}_{B}^{-1}\left(\mathbf{h}_{B}\right)\right) & =\frac{1}{N \cdot \mathrm{SNR}} \sum_{k=0}^{\infty} \sum_{i=1}^{L}\left(\frac{-1}{N \cdot \mathrm{SNR} \cdot \lambda_{i}}\right)^{k} \\
& =\frac{1}{N \cdot \mathrm{SNR}} \sum_{i=1}^{L} \frac{1}{1+\frac{1}{N \cdot \mathrm{SNR} \cdot \lambda_{i}}} \\
& =\sum_{i=1}^{L} \frac{1}{N \cdot \mathrm{SNR}+\frac{1}{\lambda_{i}}}
\end{aligned}
$$

since we have assumed that $1 /\left(N \cdot \operatorname{SNR} \cdot \lambda_{i}\right)<1$ for $i=1,2, \ldots, L$. Finally, substituting (30) in (23) yields the bound

$$
\bar{\varepsilon}_{B, L} \geq \sum_{i=1}^{L} \frac{1}{N \cdot \mathrm{SNR}+\frac{1}{\lambda_{i}}} \triangleq \bar{\beta}_{B, L} .
$$

It is worth noting that this bound depends on the statistical properties of the channel through the eigenvalues of the matrix $\mathbf{R}_{\mathbf{h}}$, whose structure is related to the shape of $R_{H}(f)$ (or, equivalently, of $P_{h}(\tau)$ ). Let us try now to simplify this bound under the assumption that the bandwidth $B$ of the sounding signal is substantially larger than the coherence bandwidth $B_{c}$ of the communication channel (wideband channel sounding). In this case we have that6 (see (19)) $\int_{f=-B-f_{2}}^{B-f_{2}} R_{H}(f) \exp \left(j 2 \pi l \frac{f}{2 B}\right) d f \cong$ $P_{h}\left(\frac{l}{2 B}\right) \cong P_{h}(0)$ for any $f_{2} \in(-B, B)$, so that $\mathbb{E}\left\{h_{B, l} h_{B, k}^{*}\right\} \cong P_{h}(0) / 2 B$ if $l=k$ and $=0$ if $l \neq k$. Then, the channel taps are uncorrelated, $\mathbf{R}_{\mathbf{h}}^{-1}=\left(2 B / P_{h}(0)\right) \mathbf{I}_{L}$, and (see (26) $)$

\footnotetext{
${ }^{6}$ This approximation is motivated by the fact that $B_{c}$ provides an indication of the width of $R_{H}(f)$ (i.e., of the frequency interval over which $R_{H}(f)$ takes on significant values). Then, if $B \gg B_{c}$, the following integral is negligibly influenced by a change in the center $\left(f_{2}\right)$ of the integration interval.
}

\begin{tabular}{|l|l|l|l|l|}
\hline & $\mathrm{E}$ & $\mathrm{G}$ & $\mathrm{U}$ & $\mathrm{TE}$ \\
\hline$B=1 / \tau_{d s}$ & $(1,5)$ & $(3,4)$ & $(1,6)$ & $(1,6)$ \\
\hline$B=10 / \tau_{d s}$ & $(1,48)$ & $(33,33)$ & $(1,61)$ & $(1,63)$ \\
\hline
\end{tabular}

Table I

VALUES OF THE COUPLE $\left(L_{1}, L_{2}\right)$ CAPTURING AT LEAST $90 \%$ OF THE OVERALL AVERAGE ENERGY OF $h_{B}(t)$.

$\mathbf{J}_{B}\left(\mathbf{h}_{B}\right)=\left(N \cdot \mathrm{SNR}+\frac{2 B}{P_{h}(0)}\right) \mathbf{I}_{L}$, so that the bound (23) becomes

$$
\bar{\varepsilon}_{B, L} \geq \frac{L}{N \cdot \mathrm{SNR}+2 B / P_{h}(0)} \triangleq \bar{\beta}_{B, L}^{(w)} .
$$

Note that $2 B / P_{h}(0) \gg 1$ because of the assumption of wideband signalling over the communication channel. Therefore, a comparison of the last result with (18) evidences that, in this scenario, a significant improvement in the quality of channel estimates should be expected if the channel estimator is endowed with a knowledge of the channel statistics.

Finally, we note that the result (32) is substantially different from the BCRB evaluated in [3, Appendix A], which refers to a discrete-time channel model in which the channel taps are independent and identically distributed random variables with a given pdf.

\section{NUMERICAL RESUlTS}

The bounds expressed by (18) and (23) (with $\mathbf{J}_{B}(\mathbf{h})$ given by (26) have been evaluated for an exponential $(\mathrm{E})$, a Gaussian (G), a uniform (U) and a truncated exponential (TE) PDP [11], so that $P_{h}(\tau)=\frac{e^{-\tau / \tau d s}}{\tau_{d s}} \mathrm{u}(\tau)$, $P_{h}(\tau)=\frac{e^{-\tau^{2} /\left(2 \tau_{d s}^{2}\right)}}{\tau_{d s} \sqrt{2 \pi}}, \quad P_{h}(\tau)=\frac{\mathrm{u}(\tau)-\mathrm{u}\left(\tau-\tau_{d s} \sqrt{12}\right)}{\tau_{d s} \sqrt{12}}$, $P_{h}(\tau)=\frac{\mathrm{u}(\tau)-\mathrm{u}\left(\tau-\tau_{M}\right)}{\tau_{0}\left(1-e^{-\tau_{M} / \tau_{0}}\right)} e^{-\tau / \tau_{0}}$ respectively, where $\mathrm{u}(\tau)$ is the unitary step function, $\tau_{d s}$ is the rms channel delay spread, $\tau_{M}$ is the maximum delay in the TE PDP and $\tau_{0}$ is another time parameter depending on $\tau_{d s}$ (see [11, eq. (16)]). In our simulations the channel bandwidths $B=1 / \tau_{d s}$ and $B=10 / \tau_{d s}$ (wideband channel sounding) have been taken into consideration. In both cases and for each of the above mentioned PDP's we have evaluated the smallest values of the parameters $L_{1}$ and $L_{2}$ ensuring that the overall average energy $\sum_{l=-L_{1}}^{L_{2}} \mathbb{E}\left\{\left|h_{B, l}\right|^{2}\right\}$ (where $\mathbb{E}\left\{\left|h_{B, l}\right|^{2}\right\}$ is given by (20) associated with the RHS of (3) is at least $90 \%$ of the overall average energy of $h_{B}(t)$ (see Table (I). Then, on the basis of such values, the couples $\left(L_{1}, L_{2}\right)=(3,6)$ and $\left(L_{1}, L_{2}\right)=(33,63)$ have been selected for $B=1 / \tau_{d s}$ and $B=10 / \tau_{d s}$, respectively, since they encompass all the cases of Table [I Fig. 2 (Fig. 3) illustrates the bounds $\beta_{B, L}$ (18) and $\bar{\beta}_{B, L}$ (23) versus the SNR for $B=1 / \tau_{d s}\left(B=10 / \tau_{d s}\right)$ and all the 


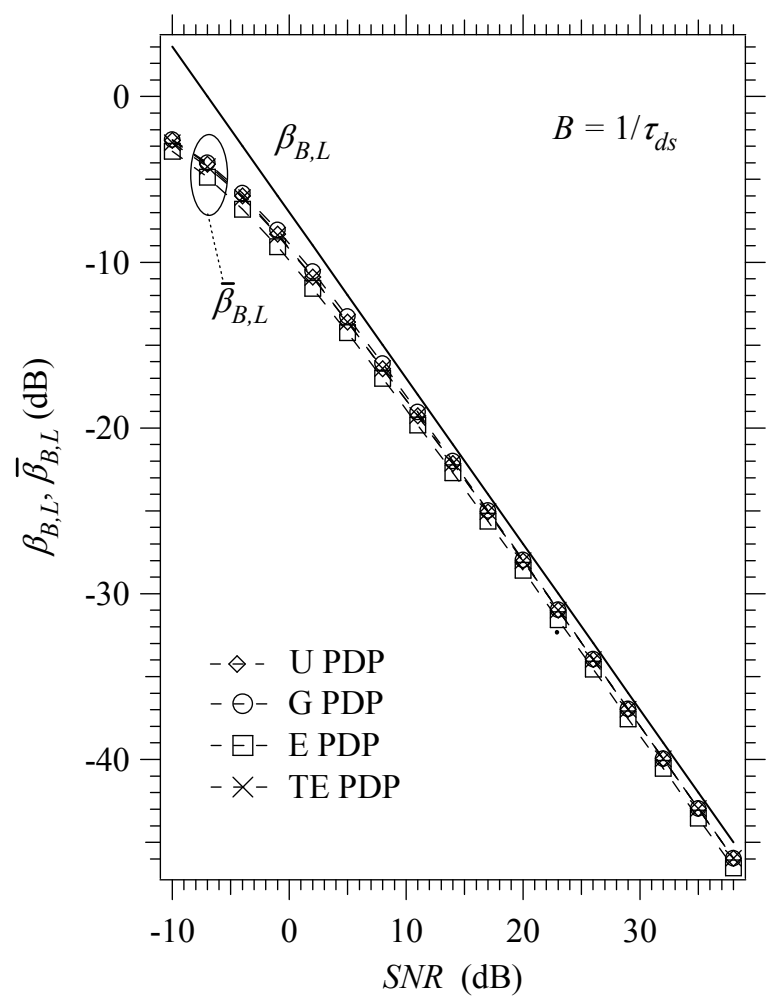

Figure 2. Performance bounds $\beta_{B, L} 18$ and $\bar{\beta}_{B, L} 23$ versus the SNR for different PDP's in the case $B=1 / \tau_{d s}$.

considered PDP's. These results show that: a) independently of the bandwidth adopted for data transmission, the impact of the availability of a priori information on the estimation accuracy of a communication channel is significant mainly at low SNR's (where the terms $\left\{1 / \lambda_{i}\right\}$ (31), not included in (18), yield a performance floor); b) the BCRB is negligibly influenced by the PDP type; c) there is a significant performance gap between the case $B=10 / \tau_{d s}$ and $B=1 / \tau_{d s}$ (this is due to the fact that the overall number of channel taps to be estimated in the latter case is substantially smaller than that of the former one). Our simulations have also evidenced that: 1) in the considered scenarios an accurate approximation of (23) is provided by eq. (31) for both values of $B ; 2$ ) eq. (32) represents a loose bound for the case $B=10 / \tau_{d s}$.

\section{Conclusions}

The problem of assessing performance limits on pilotaided channel estimation of a time-continuous frequency selective channel has been investigated. Novel bounds based on the CRB and the BCRB for TDL channel models have been derived and have been assessed for two different scenarios. The derived results shed new light on the achievable limits of pilot-aided channel estimation and the properties of optimal waveforms for channel sounding.

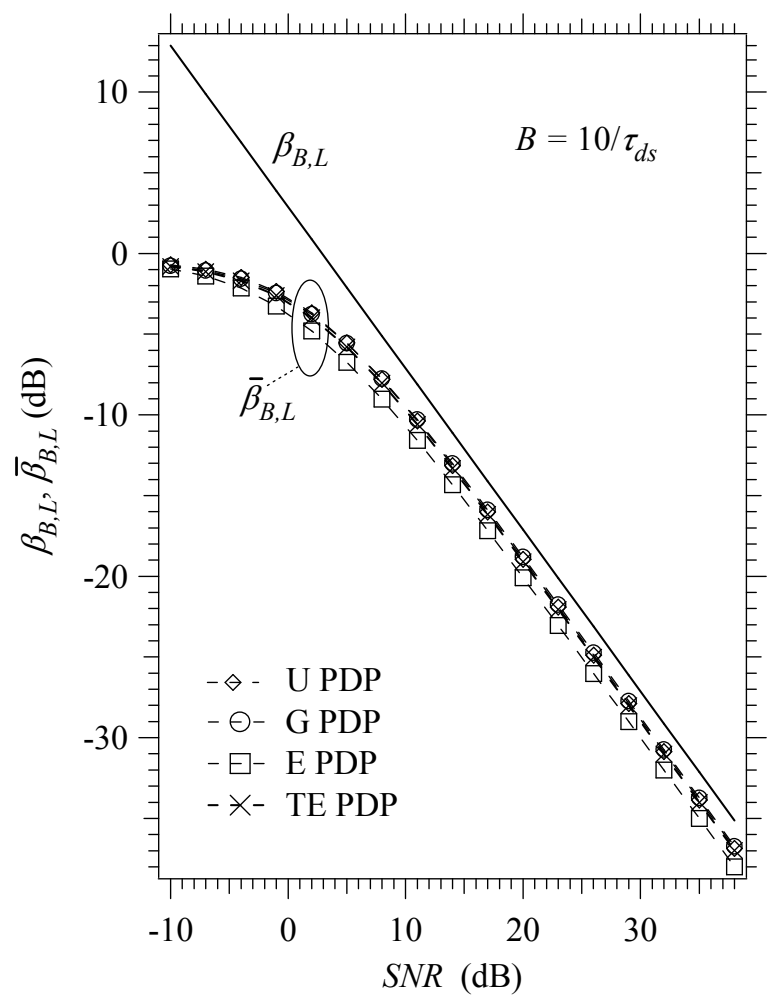

Figure 3. Performance bounds $\beta_{B, L} 18$ and $\bar{\beta}_{B, L} 23$ versus the SNR for different PDP's in the case $B=10 / \tau_{d s}$.

\section{REFERENCES}

[1] L. Tong, B. M. Sadler, and M. Dong, "Pilot-assisted wireless transmissions: General model, design criteria, and signal processing," IEEE Signal Processing Mag., vol. 21, pp. 12-25, Nov. 2004.

[2] E. De Carvalho and D. Slock, "Cramer-Rao bounds for semiblind, blind and training sequence based channel estimation," First IEEE Signal Processing Workshop on Signal Processing Advances in Wireless Communications, pp. 129-132, Apr. 1997.

[3] M. Dong and L. Tong, "Optimal design and placement of pilot symbols for channel estimation," IEEE Trans. Signal Process., vol. 50, pp. 3055-3069, Dec. 2002.

[4] P. Bello, "Characterization of randomly time-variant linear channels," IEEE Trans. Commun. Systems, vol. 11, pp. 360393, Dec. 1963.

[5] S. Kay, Fundamentals of Statistical Signal Processing: Estimation Theory, vol. I. Prentice Hall, 1993.

[6] J.-P. Delmas and H. Abeida, "Stochastic Cramer-Rao bound for noncircular signals with application to DOA estimation," IEEE Trans. Signal Process., vol. 52, pp. 3192-3199, Nov. 2004.

[7] P. Stoica and R. Moses, Introduction to Spectral Analysis. Upper Saddle River, NJ: Prentice-Hall, 1997.

[8] M. Gray, "Toeplitz and circulant matrices: A review." Available online at http://ee.stanford.edu/ gray/toeplitz.pdf

[9] H. L. V. Trees, Detection, Estimation and Modulation Theory, Part I. John Wiley \& Sons, New York, 1968.

[10] G. Strang, Linear Algebra and Its Applications. Thomson Brooks/Cole, fourth ed., 2006.

[11] E. Chiavaccini and G. Vitetta, "GQR models for multipath rayleigh fading channels," IEEE J. Sel. Areas Commun., vol. 19, pp. 1009-1018, Jun. 2001. 